\title{
The Teaching Reform and Practice of Innovative Artificial Intelligence
}

\author{
Ying Wang \\ Wuhan City Vocational College, China
}

Keywords: innovative; artificial intelligence; teaching reform; teaching practice

\begin{abstract}
Artificial intelligence is an important professional course in computer science and technology. It is a comprehensive discipline that uses computer simulation to extend human brain function. This paper aims at the problems in the teaching of artificial intelligence courses, according to the characteristics of artificial intelligence courses, puts forward a series of educational reform measures including the selection of course contents, the design of teaching methods, and the cultivation of trainees' academic abilities. The practice has proved that the implementation of the reform measures has improved the curriculum. The level of teaching effectively guarantees the quality of postgraduate training with effective teaching effects.
\end{abstract}

\section{Introduction}

Artificial Intelligence (AI) is an important professional course in computer science and technology. It is a comprehensive discipline that uses computer simulation to extend human brain function. It studies how to use computers to imitate the thinking activities that the human brain engages in reasoning, proving, identifying, understanding, designing, learning, thinking, planning, and problem solving, and solves complex problems that require human experts to deal with such as consultation and diagnosis, prediction, planning and other decision-making issues [1]. Artificial intelligence is an intersecting and comprehensive subject involving mathematics, computers, cybernetics, informatics, psychology, philosophy, and other disciplines. At present, many research fields in artificial intelligence, such as natural language processing, pattern recognition, machine learning, data mining, intelligent retrieval, robotics, and intelligent computing, are all at the forefront of information technology. Many research results have entered and influenced people.

Higher education adapting to the development of the knowledge economy should place outstanding abilities in cultivating creativity and innovation. Innovation is the life of basic research, and the teaching of institutions of higher learning is closely integrated with scientific research. Only in this way can we make a difference in cultivating students' innovative spirit. For this reason, in light of the characteristics of the artificial intelligence curriculum, we actively carry out research-based teaching, research-based learning, and improve the research and practice of university students' learning ability, practical ability and innovation ability [1]. The main contents of our teaching research and practice include three aspects: the unstructured thinking of heuristic teaching of artificial intelligence to solve problems; systemic experimental training; and graduation thesis, university student scientific research project financing plan, national university student innovation The experimental plan is connected with scientific research training. These three major aspects, which are progressive and interlocking, are a complete and innovative artificial intelligence teaching practice. Below, we will discuss these three aspects.

\section{The Current State of Artificial Intelligence Teaching}

Artificial intelligence courseware mostly uses decision theory and stochastic learning mode, which greatly simplifies the expression of the learning process. With the development of artificial intelligence technology, students' learning behaviors and training strategies have been added to the artificial intelligence system [1]. At the same time, AI technology has been applied to establish a learning advisor module (problems and skills for storing the courses to be taught). This method can control the training strategy and give students the learning content. In order to gain flexibility and 
modularity in the representation and control of curriculum knowledge, some artificial intelligence systems also use AI technology to represent training plans and strategies. For example, artificial intelligence for most programming languages is an example of this.

\subsection{Outdated teaching content}

The syllabus for the curriculum was established in the 1990s. There has been no change in more than ten years and it has lagged far behind the development of artificial intelligence technology [2]. While the teacher is basically in accordance with the syllabus to carry out the teaching, the teaching content is not updated much. Most of the curriculum is based on some traditional basic content. From the students' feedback, they are more interested in the frontier progress and the latest achievements of the discipline. Therefore, they need to guide students to actively study independently from the interest aspect. In this discipline, they not only have solid The foundation of the book also has broad knowledge.

\subsection{Irrational teaching process}

There are also many unreasonable aspects in the teaching process. Too much emphasis is placed on teachers' teaching. The students' independent thinking and practice courses are not at all. The practice link is the best way to test students' creative thinking ability. It is an essential part of training students' information collection, analysis, processing and application.

\subsection{The challenges faced by artificial intelligence teaching}

So far, most of the traditional artificial intelligences used are all programmatically pre-programmed in the courseware. Once the artificial intelligence courseware is completed, any major teaching changes will bring maintenance work. It is great inconvenience. Therefore, the existing artificial intelligence system faces many challenges. It mainly has the following problems [2]:

1) Lack of openness. The lack of openness is the biggest drawback of current artificial intelligence courseware. The user cannot make any changes to the courseware and can use only the available resources to teach according to the set route. The disadvantages are: a. The limitations of fixed content make the application of courseware narrow; $\mathbf{b}$. The set course of operation makes the teaching lack autonomy; c. The teaching is not pertinent; $\mathbf{d}$. It cannot be used at the higher starting point with the newly emerged resources.

2) There is a lack of human-computer interaction capabilities. Most of the existing artificial intelligence uses CD-ROM as a carrier of information to display the contents of the teaching materials in the form of multimedia. The teaching information is provided to learners mechanically according to a preset teaching process. The use of artificial intelligence courseware learning is completely passive. In the classroom teaching, the teacher can only operate according to the predetermined courseware flow. No matter whether the student or the teacher can participate in the teaching and learning process, human-computer interaction is not well implemented.

3) Ignore the characteristics of the course itself. Each course has different requirements in teaching, but the existing courseware ignores these different requirements completely. For example, many courses involve a large number of curves or surfaces. For some courses, it is sufficient to give a simple demonstration of these curves or surfaces, and some courses do not meet the teaching purpose. For example, when teaching algorithms for generating various curves or surfaces in computer graphics, if the process of generating these graphics can be directly and dynamically displayed in the courseware, giving full play to the advantages of computer-assisted teaching will undoubtedly make the teaching of computer graphics even easier. Attractive, thus it is greatly improving teaching efficiency.

4) Lack of teacher-student interaction. When the current artificial intelligence courseware is self-taught and operated by students, how to learn is a student's own business. Teachers cannot fully understand the situation of learners. Students cannot ask teachers for assistance when they encounter problems. Teachers and students are closed to each other and teachers and students cannot interact. Therefore, the effect of courseware is greatly reduced. At the same time, due to the lack of network 
support, most of the existing artificial intelligence courseware is run in a stand-alone environment. They cannot use the advantages of the network to quickly update the knowledge content, and certainly cannot provide convenient learning discussion space anytime, anywhere.

5) Lack of teaching strategies. In fact, the design of teaching strategies cannot be separated from the courseware development process, but the authors of courseware are often unaware of this. For example, the vast majority of the existing courseware is a single exhibition broadcast. Such courseware production is "exquisite", but it is irreversible and cannot be interactive. In fact, the use of courseware teaching is only a means rather than an objective. It is necessary to emphasize the effectiveness of courseware under the guidance of instructional design theory. The focus is on helping students learn new knowledge, master new techniques, and develop various abilities, not on the surface.

6) Lack of intelligence. The current artificial intelligence courseware system cannot provide targeted education to students of different degrees. Students' learning is passive. Students cannot selectively provide student assistance information to enable students to selectively study. For teachers, their teaching cannot be actively involved, they cannot prepare the most suitable learning content according to the student's cognitive model according to the information provided by the system, and they cannot give different modes of teaching methods and methods. Therefore, they are not intelligent.

\section{Artificial Intelligence Teaching Reform and Practice}

In order to comprehensively improve the quality of artificial intelligence teaching and the quality of students' training, students can truly learn the in-depth knowledge of artificial intelligence and have a strong practical ability to create innovations for them in the subsequent engineering practice and research process. The research results laid the foundation, it is necessary to carry out the reform of the teaching of "Artificial Intelligence" course to solve the above problems, and formulate a reasonable course content and teaching plan.

\subsection{Heuristic teaching of artificial intelligence to solve unstructured ideas}

The problems of the real world can be divided into three levels according to the degree of structuralization [3]:

1) Structured problems, a type of problems that can be described and solved by formal methods;

2) Unstructured problems, difficult to use in deterministic forms Description, mainly based on experience to solve;

3) Semi-structured problem, between the above two. In general, other courses in computer science, such as network technology, database technology, algorithm analysis and design, are all basic techniques for solving structural problems. Artificial intelligence technology is an effective technology for solving unstructured and semi-structured problems. Artificial intelligence teaching allows students to gain an understanding of the process of unstructured and semi-structured problems in the process of experiencing and understanding artificial intelligence knowledge and technology, so as to achieve the purpose of cultivating students' multi-angle thinking.

In the specific teaching of artificial intelligence, question teaching method and participatory teaching method are adopted. In the problem teaching method, knowledge modules around artificial intelligence teach students knowledge on the premise of guiding students to find various problems. In the teaching activities, attempts are made to present the artificial intelligence knowledge around practical problems, so that the problem not only becomes a prerequisite for stimulating students' thirst for knowledge, but also becomes a prerequisite for students to look forward, understand and absorb knowledge, thereby stimulating students' creative motives and creative thinking. In participatory teaching, breaking the boring, boring traditional teaching methods of artificial intelligence algorithms, trying open teaching content; questioning lectures; curriculum design without standard answers; searching literature, grouping hands-on realization of artificial intelligence algorithms and other participatory teaching methods, to develop and promote students' awareness of participation, to improve students' initiative, enthusiasm and efficiency through participatory teaching, and to 
cultivate students' hands-on and creative abilities.

\subsection{Change course teaching method}

The establishment of a classroom teaching system that is scientific, reasonable, and adaptable to the current development of artificial intelligence plays a fundamental role in enriching classroom content and improving actual teaching effectiveness.

1) Practical application learning. Research on artificial intelligence has not only stayed at the theoretical level, but has also been widely used in practical applications. However, due to its profound theories, it will cause greater difficulties for students who are actually exposed to practical applications. How to revolve around practical issues, step by step, and grasp the basic theory while solving practical problems and improving students' interest in learning [4]. This will lay a solid foundation for further study of deeper theories and practical applications, and ultimately put the knowledge of the syllabus requirements on actual practical examples, making it easier for students to absorb and master the theoretical knowledge, and at the same time, the students will be more prone to exert themselves. The initiative has deepened understanding of the focus and difficulty of the curriculum.

Under the premise of rapid development of science and technology, the speed of updating the subject knowledge of artificial intelligence has also been significantly accelerated. Due to the timeliness of publication, certain knowledge, concepts, and techniques of textbooks have lagged behind the development of disciplines [4]. This is particularly prominent in the information science disciplines, and artificial intelligence is more prominent as a forward-looking component of the current information science discipline. Therefore, under the premise of guaranteeing teaching content and teaching quality, students should be given more targeted introductions to the current development of the subject. In addition, it is necessary to inspire students' eagerness for and pursuit of innovation so that they can adapt to the characteristics of the development of science and technology so as to keep up with the changing circumstances of the discipline and lays a solid foundation for further scientific research.

2) Interactive teaching. In the classroom, students will be asked specific questions and answers for what they have learned. Starting from the key knowledge points and the appropriate content, the students will be divided into several groups to create a suitable group [5]. The academic discussion environment stimulates students' initiative thinking and divergent thinking. It provides a better way for students to understand and absorb difficulties and key knowledge points. At the same time, they should guide and pay attention to the students' responses in a timely manner and make targeted adjustments. In addition, this part should be included as a normal score in the final course assessment system to improve students' participation and enthusiasm.

3) Make full use of multimedia courseware and network resources. In general, the relevant resources provided by the network are relatively new, but duplicate or redundant content may appear. Therefore, these materials need to be collected and organized to produce rich content, reasonable structure, and ease [5]. The multimedia courseware for teaching will enable students to experience the unique charm of artificial intelligence more effectively and stimulate their curiosity and interest in the course.

The main method of processing materials is to classify and sort according to the knowledge points and the obtained file categories. On this basis, making full use of some courseware production software for processing and processing plays an important role in improving the popularity of courseware.

\subsection{Strengthen teaching in experiment}

While teaching theoretical knowledge, artificial intelligence teaching should also pay attention to practical teaching, transform abstract knowledge into images, and make intuitive experiments, so that students can truly understand the concept, nature, and research goals of artificial intelligence, thereby enhancing students' multi-perspectives [6]. The ability to think and logical reasoning, to further understand the forefront of the development of information technology, computer technology, to cultivate their interest in artificial intelligence research, stimulate the pursuit of artificial intelligence 
technology in the future.

The experimental process focuses on the key steps for students to grasp the state space to solve problems: problem representation and search strategy. The problem representation is to determine the basic information of the problem and the data structure implemented by the program. The basic information includes the initial state set, the operator set, the target detection and the path cost function [6]. The data structure can be in the form of vector, linked list, etc.; the search strategy can be divided into Blind search and heuristic search can be based on the principle of easy-to-follow, first realize breadth-first and depth-first search in blind search, and then define the valuation function to implement heuristic search. In the heuristic search implementation process, different heuristic functions can be defined: for example, the number of cards with a different state pattern and target node pattern, and the distance between the target position and the distance between the target position and the distance between the target position and the target, and accurately compare the meaning of the heuristic function. Through experiments, the trainees deepened their understanding of the theoretical knowledge taught in the classroom and were able to skillfully use the state space approach to solve practical problems and improve their engineering practice capabilities.

\section{Summary}

The era of artificial intelligence has come. In the next decade, artificial intelligence will have a vast space in the teaching of universities and will become the frontier for the next innovation, competition, and efficiency improvement. We should seize the opportunity and timely reform to make universities in the information age artificial. Intelligent education keeps pace with the times and cultivates more qualified personnel who can adapt to the development of the information society.

\section{References}

[1] J.P. Zhang, Thinking about artificial intelligence education, Audio-visual education research, 2003, vol.1. pp.24-28.

[2] W.S. Wang, The principle of artificial intelligence and its application, China Educational Technology Equipment, 2007, pp.48-51.

[3] J.T. Cong and J.A. Liu, The role of artificial intelligence education in capacity development and reform assumptions, Computer age, 2006, vol.9. pp.66-69.

[4] J.W. Bai and J.M. Bi, A Preliminary Study on the Teaching Reform of Artificial Intelligence, China Educational Technology and Equipment, 2010, vol36, pp.43-44.

[5] Ch.H. Xiao and L.L. Hu, On the Cultivation of Postgraduate Academic Competence, Degree and Graduate Education, 2006, vol.9, pp.1-5.

[6] J.H. Zhou, Artificial Intelligence Learning Counseling and Experimental Guidance, Electrification Education Research, 2008, vol.4, pp.20-24. 